\title{
In vitro assessment of a novel additive manufactured titanium implant abutment
}

\section{Les Kalman}

Assistant Professor, Restorative Dentistry, Chair, Dental Outreach, Schulich School of Medicine \& Dentistry, Western University, 1151 Richmond Street, London, Ontario, Canada

Correspondence:

Schulich School of Medicine \& Dentistry

Western University

1151 Richmond Street

London, Ontario, Canada

N6A-3K7

ljkalman@icloud.com

Kalman L. In vitro assessment of a novel additive manufactured titanium implant abutment. J Clin Exp Dent. 2021;13(2):e99-103.

Received: 29/05/2020

Accepted: $23 / 09 / 2020$

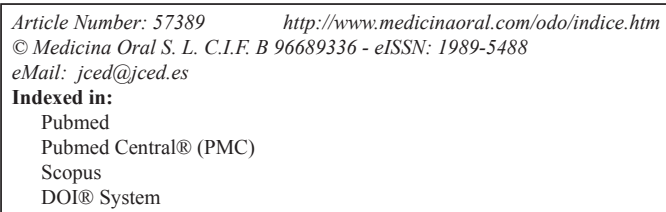

\begin{abstract}
Background: Dental implant temporization remains a clinical challenge. A novel abutment simplifying the procedure was developed, but conventional fabrication was costly and unpredictable. A workflow was developed to fabricate the novel abutment using additive manufacturing. This in vitro investigation assessed the additive manufactured (AM) novel abutments to conventionally manufactured (CM) abutments.

Material and Methods: The AM abutments were fabricated in dental-grade titanium (Ti-6Al-4V) using Selective Laser Melting and were post-processed. The CM abutments were milled and subsequently laser welded manually. Pin strength of the abutments was assessed using a universal loading machine. Torque was measured by tightening the $\mathrm{AM}$ and $\mathrm{CM}$ abutments into dental implants within artificial bone.

Results: Average pin strength was $364.4 \mathrm{~N}$ for the $\mathrm{AM}$ abutments and $62.5 \mathrm{~N}$ for the $\mathrm{CM}$ abutments. Average torque was $49.9 \mathrm{Ncm}$ for the $\mathrm{AM}$ abutments and $62.9 \mathrm{Ncm}$ for the $\mathrm{CM}$ abutments. AM abutment's pin strength was higher than the CM abutments. CM abutments measured a higher torque than the AM abutments.

Conclusions: Additive manufacturing with titanium using SLM provided an alternative fabrication pathway of a novel implant abutment. The AM approach was cost-effective, predictable, efficient and demonstrated pin strength and torque suitable for temporization procedures in implant dentistry.
\end{abstract}

Key words: Abutment, dental implant, temporization, medical device, prototype design, additive manufacturing, $3 D$ printing, titanium.

\section{Introduction}

Implant-supported restorations are a well-recognized esthetic and functional solution for partially edentulous patients $(1,2)$. In the interim phase of treatment, temporary restorations are required to restore gingival health $(2,3)$, while providing esthetic and functional benefits to the patient (1). Despite these advantages, the use of temporaries present a challenging situation (4). With high potential for clinical failure $(2,5,6)$ and a demand for optimal esthetics (7), considerable scientific interest has been focused on refining the components and processes for predictable implant temporaries (8). 
Failure of implant-supported restorations may be directly related to the component design of implant temporaries (9). Cho and colleagues (10) reported that many temporary prostheses applied external stressors that initiated soft-tissue inflammation and inhibited osseointegration. Poorly fitted removable partial dentures can induce undesired forces and resultant stresses in the healing cap and/or implant body (11). These undesired stresses can eventually lead to failure of the implant-supported restorations (12). Therefore, further research focused on temporary abutment designs that minimize these forces is required.

A novel abutment and process for temporization (U.S. Patent No. 12/668832) has been developed (Research Driven, Ontario, Canada) that addresses the functional, aesthetic, and financial requirements of temporary prosthesis and may be considered as an alternative option (13).

The novel abutment demonstrated advantageous characteristics for its potential use (13), but the production was unpredictable and costly with traditional machining and laser welding, due to the size and intricate geometric features (Fig. 1A). A workflow was recently developed to apply additive manufacturing using titanium to fabricate the novel abutment (Fig. 1B) (14).

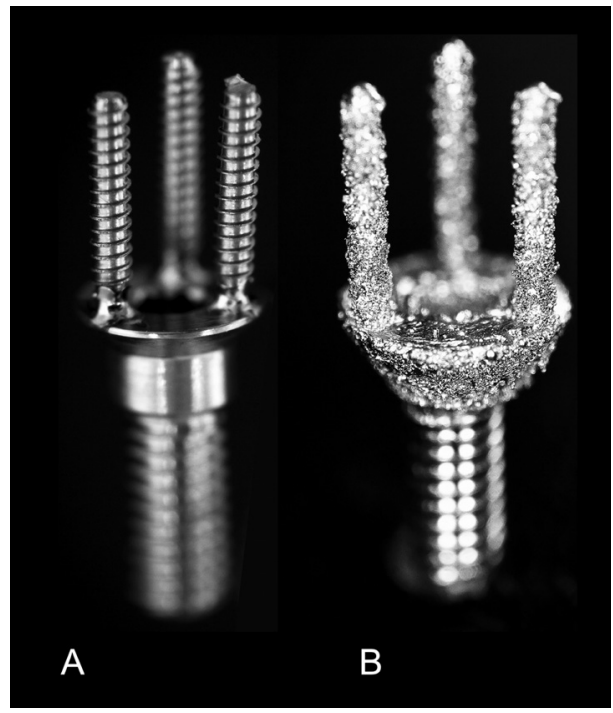

Fig. 1: A. Conventionally manufactured novel abutment. B. Additive manufactured novel abutment.

This in vitro investigation assessed the torque and pin strength of the additive manufactured (AM) novel abutment against that of a conventionally manufactured (CM) abutment. The investigation would provide important data to determine if the abutment could tolerate (1) tightening within an implant body and (2) the process of temporization.

\section{Material and Methods}

The CM abutments were fabricated by utilizing a $6 \mathrm{~mm}$ diameter, $1.5 \mathrm{~mm}$ in height, titanium regular neck closure cap (Straumann, Zurich, Switzerland) and $0.60 \mathrm{~mm}$ diameter titanium dentine pins (Fairfax Dental, London, England). The pins were manually laser welded to the closure cap with titanium laser wire (Biogenic Technical Institute, Utica, NY) using a magnified laser welder (LaserStar Technologies, Orlando, Florida). The CM abutments were fabricated through a commercial dental laboratory (Expertec Dental Lab, Westland, Michigan). The AM abutment was developed through a novel workflow (14). The digital design was optimized for additive manufacturing and the abutment was printed in dental-grade titanium (Ti-6Al-4V) (ADEISS, London, Canada). The abutments underwent post-processing, which included final thread tapping to accentuate thread geometries, heat treatment for relieving thermal stresses and strengthening, and bead blasting for a smoother finish.

The CM and HM abutments were evaluated for fit and suitability with a dental implant analogue through visual, tactile and radiographic assessment by an experienced clinician.

-Assessment of Pin Strength

The novel abutment has a unique process for temporization (13). The abutment has three pin projections that act to retain the temporary crown. Accordingly, the strength of the pins required assessment. The temporization technique is unique, with limited physical data, and this assessment (Fig. 2A) was developed similar to other pin testing protocols.

Five custom jigs were fabricated to facilitate the assessment (Fig. 2A). Jigs were fabricated from light cured resin (Dentsply, York, USA) with an embedded implant analogue (Straumann, Zurich, Switzerland). The resin jigs were fabricated to fit within the metal housing unit of the universal loading machine (Instron, Norwood, MA). Twenty-five [25] CM abutments and twenty-five [25] AM abutments were sequentially placed into the implant analogue and torqued to $25 \mathrm{Ncm}$. The jig was then inserted into a universal loading machine. An axial load, perpendicular to the abutment platform, was generated at a maximum cross head speed of $0.5 \mathrm{~mm} / \mathrm{min}$ until mechanical failure of the abutment occurred. The corresponding value was then measured and recorded.

-Assessment of Torque

Assessment of torque was completed with a modified torque measurement protocol (Fig. 2B). Fifty [50] dental implant analogues (Straumann, Zurich, Switzerland) were placed into artificial bone blocks (Sawbones: Pacific Research Laboratories, Vashon, Washington) by an experienced clinician. Twenty-five [25] CM abutments and twenty-five [25] AM abutments were threaded into the implant bodies. The bone blocks were placed into a custom jig. The abutments were then tightened with 


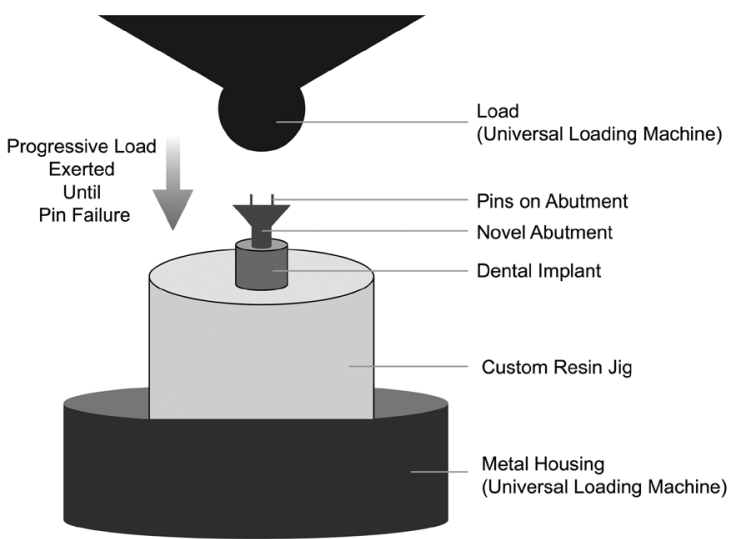

A

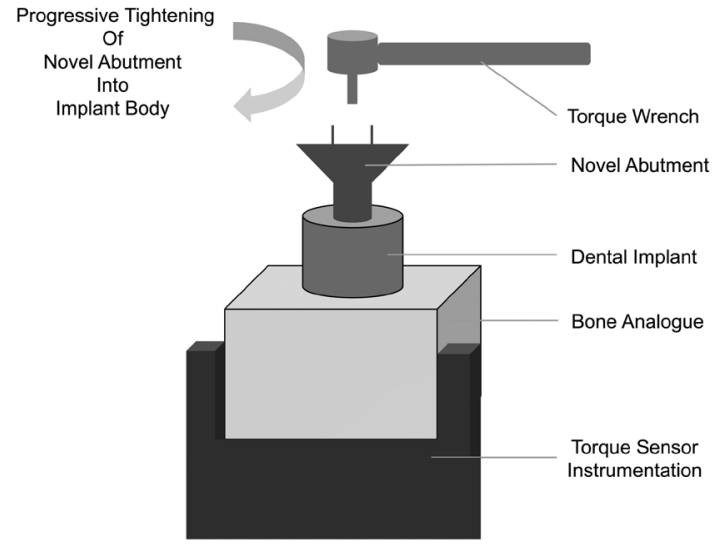

B

Fig. 2: A. Diagrammatic representation for the assessment of pin strength. B. Diagrammatic representation for the assessment of torque. standard torque wrenches (Straumann, Zurich, Switzerland; AB Dental Devices Ltd., Ashdod, Israel). Torque was measured by a torque sensor (AMTI 6-DOF; Advanced Mechanical Technology Inc., Watertown, MA) secured at the base of the bone block (Figure 2B). Torque was continuously measured during tightening, by the sensor, and the maximum torque for each abutment was recorded (Instron WaveMatrix Software; Instron, Norwood, MA).

\section{Results}

Figure 3 illustrates the data from the assessment of pin strength of novel abutments. The maximum axial load that the pin projections on the abutment could tolerate (i.e. fracture resistance), before breakage or bending, was recorded. The average pin strength for AM abutments was $364.4 \mathrm{~N}$ and for CM abutments was $62.5 \mathrm{~N}$. Minimum and maximum pin strengths for the AM abutments were $278.3 \mathrm{~N}$ and $468.9 \mathrm{~N}$, respectively. Minimum and maximum pin strengths for the $\mathrm{CM}$ abutments were $12.1 \mathrm{~N}$ and $146.9 \mathrm{~N}$, respectively.

Figure 4 illustrates the data from the assessment of torque of novel abutments. The maximum torque of the abutment into the implant body was recorded. The average torque for $\mathrm{AM}$ abutments was of $49.9 \mathrm{Ncm}$ and for CM abutments was $62.9 \mathrm{Ncm}$. Minimum and maximum torque for the AM abutments was 34.1 and $83.1 \mathrm{Ncm}$, respectively. Minimum and maximum torque for the $\mathrm{CM}$ abutments was 48.9 and $93.1 \mathrm{Ncm}$, respectively.

\section{Discussion}

The strength of the pins for the CM abutments were relatively low and most likely related to the unpredictability

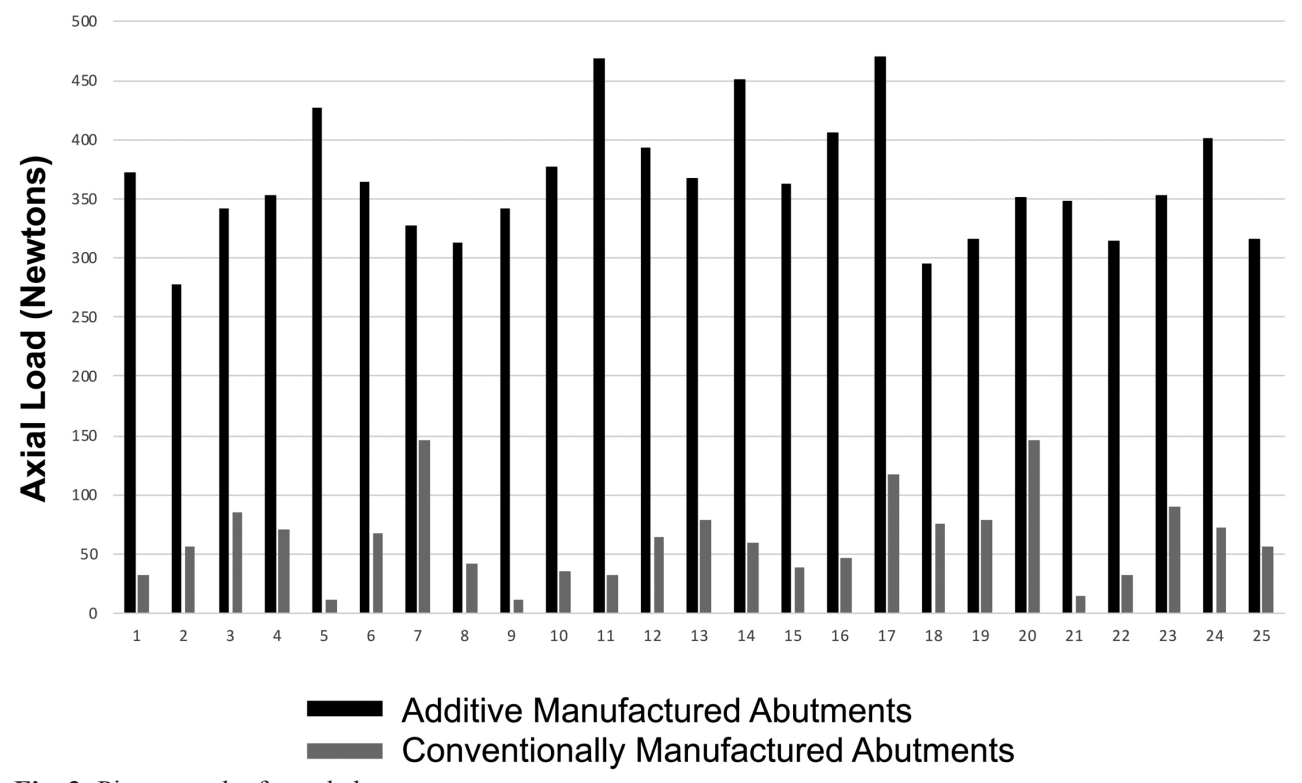

Fig. 3: Pin strength of novel abutments. 


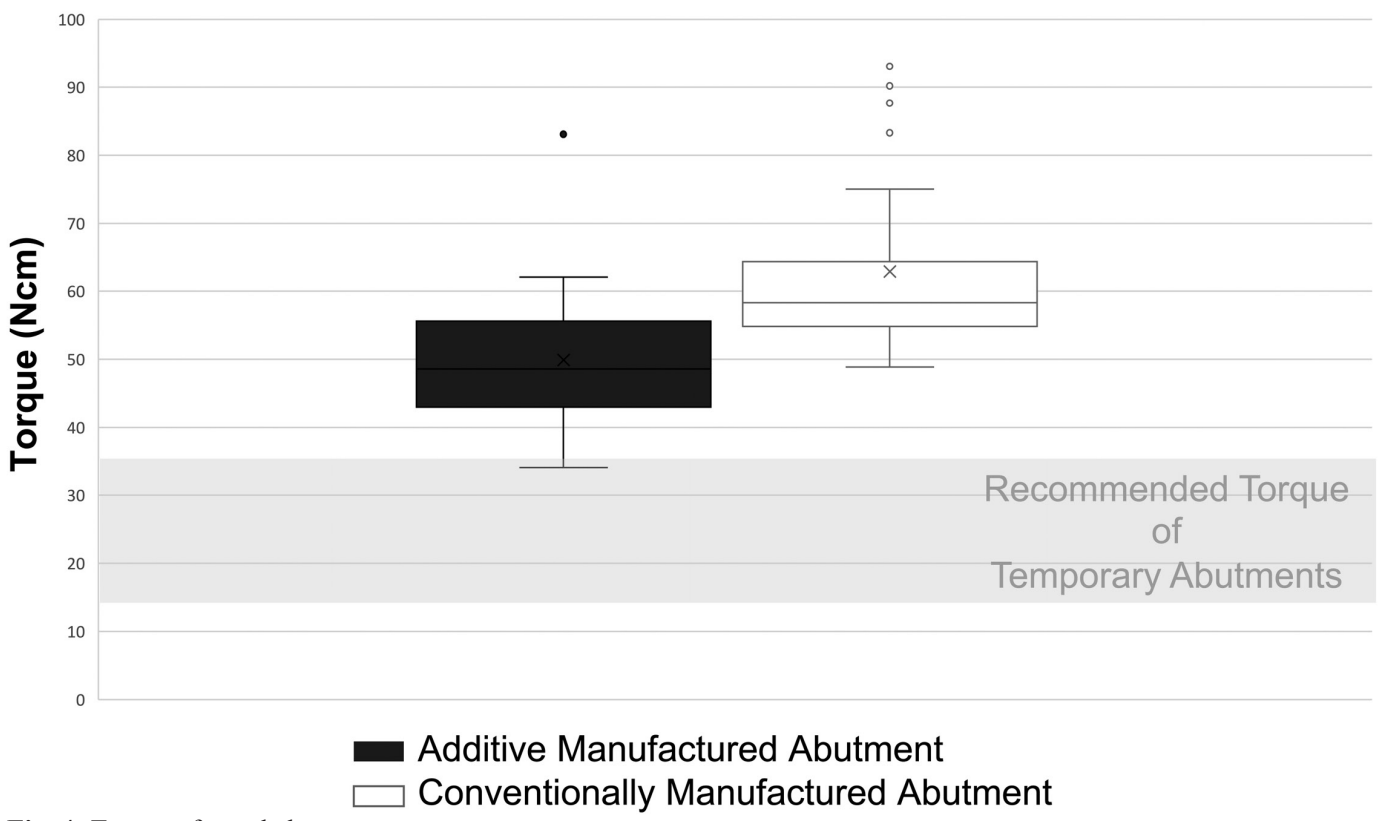

Fig. 4: Torque of novel abutments.

of manually welding titanium to titanium. The author had significant difficulty finding a commercial lab that would complete the welding, due to its complexity. The AM abutments had a relatively higher pin strength. This is most likely related to the SLM process and the 30-50 micrometer layer build.

The goal of the pin strength assessment was to estimate if the pins, and the abutment, could tolerate the temporization process. There have been no studies quantifying temporization. The author inferred that the lowest AM pin strength value $(278 \mathrm{~N})$ is approximately the force required for the extraction of a single rooted tooth (15). The temporization process would be a fraction of that value, and pin strength would be suitable for the process. Masticatory forces would not be relevant in the evaluation of the abutment, as the temporary prosthesis would be reduced out of occlusion.

The assessment of torque generated a wide range of values for both the AM and CM abutments (Fig. 4). This may be attributed to the measurement protocol. The implant analogues were placed into bone analogues. As the abutments were tightened, some of the implant bodies rotated within the bone analogue, causing inaccurate measurements.

The CM abutments had relatively higher torques than the AM abutments. This was most likely attributed to a problem with the abutment design, as the torque wrench was slipping within the receptacle of the abutment, causing inaccurate measurements. A design modification is required to incorporate a deeper receptacle or a modified geometric shape to prevent the wrench from slipping when tightening.
The lowest torque for the AM abutment was $34.1 \mathrm{Ncm}$. Technical information (16) recommends the torqueing of a metal temporary abutment should range from 15 to 35 $\mathrm{Ncm}$. The AM abutments torque values were within this range, except for one value. The data suggests that the AM abutments could be utilized for temporization procedures, as long as they are tightened at the lower limit of the recommendation, at approximately $15-25 \mathrm{Ncm}$.

The cost to manufacture the CM abutment was approximately \$225 CDN/unit. This workflow required several weeks, to acquire the materials and for outsourcing the laser welding. Approximately $50 \%$ of the CM abutments could not be used for testing, due to variations in welded pin orientation, rendering many units useless. In contrast, the AM abutments required about one week for fabrication, with a cost of approximately \$13 CDN/unit. Remarkably, $100 \%$ of the abutments could be utilized for testing.

There were many variables that could have impacted the results. Variations in the position and angulation of the welded pins on the $\mathrm{CM}$ abutment, the quality of the laser weld and the angulation of the implant in the custom jig, could have affected pin strength testing. Testing protocols could have impacted the torque assessment, as previously mentioned. Further samples and testing are required to reevaluate torque with a modified abutment design.

Based on this limited investigation, the additive manufactured novel abutment seems suitable as a temporary abutment option. Additive manufacturing with titanium using SLM, provides a predictable, cost-effective, efficient and customizable approach for the fabrication of a novel dental implant abutment. 


\section{References}

1. Lata $\mathbf{J}$ and Parmar M. Placement of single tooth implant in the healed socket with immediate temporization: clinical study. Contemp Clin Dent. 2012;3:412-415.

2. Ryu HS, Namgung C, Lee JH, Lim YJ. The influence of thread geometry on implant osseointegration under immediate loading: A literature review. J Adv Prosthodont. 2014;6:547-554.

3. Huang HL, Tsai MT, Su KC, Li YF, Hsu JT, Chang CH, et al. Relation between initial implant stability quotient and bone-implant contact percentage: An in vitro model study. J Oral Maxillofac Surg Med Pathol. 2013;116:356-361.

4. Weber HP, Sing T. Provisional Restorations in Implant Dentistry: Considerations for balancing esthetics and tissue healing in the maxillary anterior. Inside Dentistry. 2014;10.

5. Goodacre CJ, Bernal G, Rungcharassaeng K, Kan JY. Clinical complications with implants and implant prostheses. J Prosthet Dent. 2003;90:121-132.

6. Fugazzotto PA. Success and failure rates of osseointegrated implants in function in regenerated bone for 72 to 133 months. Int J Oral Maxillofac. Implants. 2005;20:77-83.

7. Mijiritsky E, Mardinger O, Mazor Z, Chaushu G. Immediate provisionalization of single-tooth implants in fresh-extraction sites at the maxillary esthetic zone: Up to 6 years of follow-up. Implant Dent. 2009;18:326-333.

8. Joda T, Ferrari M, Braegger U. A digital approach for one-step formation of the supra-implant emergence profile with an individualized CAD/CAM healing abutment. J Pros Res. 2016;60:220-223.

9. Magne P, Silva M, Oderich E, Boff LL, Enciso R. Damping behaviour of implant supported restorations. Clin Oral Implants Res. 2013;24:143-8.

10. Cho SC, Shetty S, Froum S, Elian N, Tarnow D. Fixed and removable provisional options for patients undergoing implant treatment. Compendium. 2007;28:604-609.

11. Bural C, Buzbas B, Ozatik S, Bayraktar G, Emes Y. Distal extension mandibular removable partial denture with implant support. Eur. J. Dent. 2016;10:566-570.

12. Levin L. Dealing with dental implant failures. J Appl Oral Sci. 2008;16:171-175.

13. Kalman L, Estafanos L. Implant Provisionals Utilizing a Novel Abutment: Assessing Quality, Efficiency and Stability. Int J New Tech Eng. 2017;4:37-47.

14. Kalman L, Hosein Y, Chimel T. Workflow development of a 3D printed novel implant abutment. 3D Printing \& Additive Manufacturing. 2019;6:234-237.

15. MacGregor AJ, Tomlinson GE. An apparatus for measuring the forces of dental extraction. British Journal of Oral Surgery. 1980;17:7176.

16. Institut Straumann AG (2018) Straumann Technical Information. 2018:30. Basel, Switzerland.

\section{Acknowledgments}

The author would like to thank the following for their support and commitment. Dr. Yara Hosein, for the assistance with the grant proposal, HM development and testing. Tom Chimel, for the development of the HM abutment. Dr. Elham Vakili, for assistance with the results and statistics. Dr. Amin Rizkalla for the use of his equipment and his assistance with the testing. Dr. Vishal Patel for assisting with the data collection. Aaron O'Donnell and Straumann Canada Ltd. for product support. Schulich Dentistry, for the Schulich Dentistry Research Opportunity Program (student support) and the Internal Research Grant, to assist with the funding. To the International Congress of Oral Implantologists, for partial funding by the Implant Dentistry Research \& Education Foundation.

\section{Ethics}

No ethics approval was required with this in vitro study.

\section{Sources of Funding}

Funded, in part, by the (1) Implant Dentistry Research \& Education
Foundation, a division of the International Congress of Oral Implantologists (2) Schulich Dentistry Internal Research Grant (3) Dentistry Research Opportunity Program and (4) Straumann Canada Ltd.

\section{Authors' contribution}

Sole authorship.

\section{Conflict of Interest}

The benefits accruing to the author from a commercial or industrial party will be applied to a research fund, non-profit institution or other organization with which the author is associated. The author is the co-inventor of the medical device. 